\title{
Comparative microscopic analysis of nail clippings from patients with cutaneous psoriasis and psoriatic arthritis*
}

\author{
Gabriela Poglia Fonseca ${ }^{1}$ \\ Gabriela Seidel $^{3}$
}

\author{
Betina Werner ${ }^{2}$ \\ Henrique Luiz Staub ${ }^{4}$
}

DOI: http:/ /dx.doi.org/10.1590/abd1806-4841.20175056

\begin{abstract}
BACKGROUND: The nail involvement in psoriasis is related to psoriatic arthritis and may represent a predictor of the disease.

Овјестіves: To analyze, through nail clipping, clinically normal and dystrophic nails of patients with cutaneous psoriasis and psoriatic arthritis.

MethoDs: This is a cross-sectional multicenter study, conducted between August 2011 and March 2012. Patients were divided into four groups: patients with cutaneous psoriasis and onychodystrophy, patients with cutaneous psoriasis and clinically normal nails, patients with psoriatic arthritis and onychodystrophy and patients with psoriatic arthritis and clinically normal nails. We calculated NAPSI (Nail Psoriasis Severity Index) of the nail with more clinically noticeable change. After collection and preparation of the nail clipping, the following microscopic parameters were evaluated: thickness of the nail plate and subungual region, presence or absence of parakeratosis, serous lakes, blood, and fungi.

RESULTS: There were more layers of parakeratosis $(\mathrm{p}=0.001)$ and a greater thickness of the subungual region in patients with cutaneous psoriasis and onychodystrophy $(p=0.002)$. Serous lakes were also more present in the same group $(p=0.008)$ and in patients with psoriatic arthritis and normal nails $(p=0.047)$. The other microscopic parameters showed no significant difference between normal and dystrophic nails or between patients with psoriatic arthritis or cutaneous psoriasis.

STUDY LIMITATIONS: Small sample size and use of medications.

CONCLUSIONS: Nail clipping is a simple and quick method to assess the nails of patients with nail psoriasis although does not demonstrate difference between those with joint changes or exclusively cutaneous psoriasis.
\end{abstract}

Keywords: Arthritis, psoriatic; Pathology; Psoriasis; Nails

\section{INTRODUCTION}

Psoriatic arthritis occurs in $5 \%$ to $40 \%$ of patients with psoriasis, causing significant morbidity and being responsible for progressive joint destruction. ${ }^{1,2}$ Nail dystrophy may be associated with all clinical forms of psoriasis, and may precede or not the cutaneous lesions, preferentially affecting the fingers. ${ }^{3,4}$ However, there seems to be a greater severity of nail involvement in patients with psoriatic arthritis, especially when there is involvement of the distal interphalangeal joint and enthesitis. ${ }^{1,5}$

Nail apparatus of patients with psoriatic arthritis has already been investigated by means of imaging examinations, and histological studies of cadavers have already been performed, but the nails have not previously been studied by the nail clipping method. ${ }^{6}$ This method is simple, quick and painless: the nail is cut into its distal and free portion, and the resulting fragment is pro- cessed so that it is possible to cut it on the microtome to make a slide for examination under a microscope. The greatest indication of nail clipping is in the diagnostic elucidation of onychomycosis, where the sensitivity of the test can overcome the direct mycological examination and the culture of fungi. ${ }^{7-9}$ Lately, this method has begun to be explored for better investigation of inflammatory nail diseases. ${ }^{10,11}$

In this study, the dystrophic nails of patients with psoriasis were analyzed microscopically, comparing the findings between patients presenting with arthritis and those with cutaneous psoriasis only. NAPSI (Nail Psoriasis Severity Score) was used to compare clinical parameters between the two groups. ${ }^{12,13}$ Clinically normal nails of patients with psoriatic arthritis or exclusively cutaneous psoriasis were used as control groups.

Received on 19.08.2015.

Approved by the Advisory Board and accepted for publication on 05.03.2016.

Study conducted at Hospital de Clínicas de Curitiba, Universidade Federal do Paraná (HC-UFPR), and at Hospital São Lucas, Pontifícia Universidade Católica do Rio Grande do Sul (PUCRS) - Porto Alegre (RS), Brazil.

Financial support: None.

Conflict of interest: None.

Private Clinic - Vacaria (RS), Brazil.

Dermatology and Pathology Departments of the Universidade Federal do Paraná (UFPR) - Curitiba (PR), Brazil.

Private Clinic - Campo Largo (PR), Brazil.

Department of Rheumatology of the Pontifícia Universidade Católica do Rio Grande do Sul (PUCRS) - Porto Alegre (RS), Brazil.

(C2017 by Anais Brasileiros de Dermatologia 


\section{METHODS}

A cross-sectional study was conducted, including patients diagnosed with psoriatic arthritis in follow-up at the Rheumatology Outpatient Clinic of the Pontifícia Universidade Católica do Rio Grande do Sul, and patients with exclusively cutaneous psoriasis, followed at the Dermatology Clinic of the Hospital de Clínicas de Curitiba of the Universidade Federal do Paraná, conducted in the period between August 2011 and March 2012. The study was previously approved by the Research Ethics Committee of both institutions, in compliance with Resolution CNS/MS 196/96.

The subjects were divided into four groups: patients with cutaneous psoriasis and onychodystrophy (PO group), patients with cutaneous psoriasis and clinically normal nails (P group), patients with psoriatic arthritis and onychodystrophy (PAO group), and patients with psoriatic arthritis and clinically normal nails (PA group).

Diagnosis of psoriatic arthritis was established by a rheumatologist by means of the 2006 CASPAR (Classification of Psoriatic Arthritis Study), with sensitivity of $91-100 \%$ and specificity of $97-99 \%{ }^{14}$ The diagnosis of cutaneous psoriasis was established by a dermatologist.

Exclusion criteria were high clinical suspicion of onychomycosis or other causes of nail dystrophy (as an association with nail lichen planus or trauma, for example), as well as other inflammatory arthropathies that did not meet criteria for the diagnosis of psoriatic arthritis.

After reading and signing of the informed consent by the patients, data were collected such as age, gender, duration of illness and medications used. Subsequently, the NAPSI of the nail with the highest clinically visible change was calculated. The most involved nail was divided into imaginary lines, horizontally and vertically, in four quadrants. In each quadrant, the presence or absence of nail matrix changes (pitting, leuconychia, hemorrhage in the lunula and nail crumbling) and of nail bed (onycholysis, subungual hyperkeratosis, oil spotting in water, and splinter hemorrhage) were evaluated. Each matrix and nail bed change received a score of zero to four, according to the committed quadrants. After evaluation of the eight nail parameters, a score from zero to 32 was considered for NAPSI. ${ }^{12,13}$

At least one fragment of the clinically most dystrophic nail of each patient was harvested, and a greater number of samples were collected for availability. When nails were clinically normal, the nail fragment was collected systematically from the ring finger.

Nail clipping was performed by cutting the distal portion of the free edge of the nail plate with a minimum of $5 \mathrm{~mm}$ in length and $2 \mathrm{~mm}$ in width, taking care not to result in discomfort to the patient. ${ }^{711}$ The material was stored in sterile vials containing formalin (10\% formalin solution). The technical processing of the nails for making the microscopic preparations is described in detail in another publication. ${ }^{15}$ The slides were stained with hematoxylin-eo$\sin (\mathrm{HE})$ and PAS with digestion (PAS-CD). The following microscopic parameters were evaluated: thickness of the nail plate and the subungual region (measured in millimeters with appropriate ruler), presence or absence of parakeratosis (nuclei of corneocytes of the subungual region), with annotation of the number of layers, presence of neutrophils, serous lakes, blood and fungi. Microscopic analysis was performed blindly by the dermatopathologist (BW), without previous knowledge regarding the group which sample belonged to (if the patient had arthritis or not).

Statistical analyzes were performed using the STATISTICA 7.0 and ACTION 1.0 programs. Methodologies used for the evaluation of the categorical variables, with nominal or ordinal measurements, were the chi-square test or Fisher's exact test, in the impossibility of applying the first test. For the quantitative variables, the Mann-Whitney test (when two groups were compared) and Kruskal-Wallis test (when more than two groups were compared) were applied. In the existence of statistical significance, the complementary comparisons were performed through the test of Minimum Significant Difference (msd). The level of significance applied to all tests was 0.05 .

\section{RESULTS}

We evaluated 97 patients. Of these, $41(42 \%)$ had psoriatic arthritis and 56 (57\%) exclusively cutaneous psoriasis. Onychodystrophy was detected in 51 patients (52\%). Participants were 56 women and 41 men, ranging in age from 16 to 87 years (median = 52 , Interquartile Range-IQR $=23$ ). The duration of the disease was between 3 months and 49 years (median $=11, I Q R=15$ ). Twenty patients presented psoriatic arthritis and onychodystrophy (PAO group); 21 had psoriatic arthritis and clinically normal nails (PA group); 31 patients, exclusively cutaneous psoriasis and onychodystrophy (PO group); and 25, exclusively cutaneous psoriasis with normal nails (P group). Methotrexate was the most used medication among patients $(73.1 \%)$. Table 1 shows the global clinical characteristics of the groups studied.

Among all patients with onychodystrophy, NAPSI scores ranged from 1 to 14 (median $=5, \mathrm{IQR}=3.5$ ). Main alterations were pitting and onycholysis (55\%), followed by leukonychia (29\%), oil spot in water $(27 \%)$, subungual hyperkeratosis and splinter hemorrhage (19\% each), onychorrhexis (13\%), crumbling (9\%) and Beau's lines $(2 \%)$. In the group of patients with psoriatic arthritis, the median NAPSI was 6 (2-14) and in those without arthritis, the median was 5 (1-12). There was no statistical difference between the NAPSI values of the patients with or without arthritis $(p=0.300)$.

Overall, the thickness of the nail plate varied between 0.2 and $0.85 \mathrm{~mm}$. In those patients with onychodystrophy, the thickness of the nail plate was between 0.26 and $0.85 \mathrm{~mm}$ (median $=0.4$, IQR $=0.19$ ), while in patients with clinically normal nails it ranged between 0.2 and $0.8 \mathrm{~mm}$ (median $=0.39, \mathrm{IQR}=0.19$ ). When evaluating patients with cutaneous psoriasis and psoriatic arthritis, there was no difference in the thickness of the nail plate in those with altered nails $(p=0.201)$ and those with clinically normal nails $(p=0.878)$.

Subungual thickness ranged from 0 to $0.7 \mathrm{~mm}$ in general. There was a statistically significant difference between the groups $(p=0.019)$ in the comparison between patients with cutaneous psoriasis, with or without altered nails $(p=0.002)$, and between patients with psoriasis and psoriatic arthritis with onychodystrophy $(\mathrm{p}=0.017)$.

Regarding the number of corneocytes detected in the subungual region (layers of parakeratosis), all groups presented from zero up to 53 layers of parakeratosis. In general, patients with dys- 
trophic nails had a mean of 7.5 layers of cells (maximum $=36$, minimum = zero, median $=5, \mathrm{SD}=8.16, \mathrm{IQR}=10$ ), while among those with clinically normal nails the mean number of parakeratosis layers was lower: 2.2 (maximum $=7$, minimum $=0$, median $=2.0, \mathrm{SD}=$ $2.02, \mathrm{IQR}=3)$, a statistically significant finding $(\mathrm{p}=0.001)$. When the $\mathrm{PO}$ and $\mathrm{PAO}$ groups were compared, that is, in the comparison between patients with and without arthritis, there was no statistically significant difference in this measure $(\mathrm{p}=0.383)$.

Neutrophils were seen in $11.7 \%$ of the nails of patients with onychodystrophy and $2 \%$ in the group with clinically normal nails. These cells were found similarly between patients with psoriatic arthritis and those without joint involvement $(p=0.561)$.

Serous lakes were found in $47 \%$ of patients with onychodystrophy versus $32 \%$ of those with clinically normal nails. In those with clinically normal nails, there was more evidence of serous lakes in the psoriatic arthritis group $(\mathrm{p}=0.047)$ as well as among patients with cutaneous psoriasis, where serous lakes were more present in those with onychodystrophy ( $p=0.008)$.

Blood was observed in 6 cases (11.7\%) of clinically abnormal nails and in 2 cases (4.3\%) of clinically normal nails. Statistically, there was no difference in the number of patients with onicodystrophy with arthritis from those with psoriasis alone $(p=0.230)$.

Fungi were seen in $21.5 \%$ of cases with onychodystrophy and in $10.8 \%$ of cases in which the nails were clinically normal. Only one case presented hyphae in the subungual region, being considered onychomycosis. In other cases, there were yeast forms only in the subungual region and rarely in the external surface or in the nail plate. These cases were considered as opportunism or contamination. There was no statistically significant difference between all the groups studied ( $p=0.333$ ), as there was no difference when patients with onychodystrophy with arthritis were compared with patients only with cutaneous form of psoriasis $(p=0.362)$.

TABLE 1: Clinical variables studied. Statistical comparison data between patients with and without onychodystrophy

\begin{tabular}{llll}
\hline & $\begin{array}{l}\text { Patients with } \\
\text { onychodystrophy (PAO + PO) }\end{array}$ & $\begin{array}{l}\text { Patients with normal } \\
\text { nails (PA + P) }\end{array}$ & p value \\
\hline Age (years) & $53(19-87)$ & $55(16-82)$ & 0.666 \\
Women & $22(43.1 \%)$ & $34(73.9 \%)$ & 0.002 \\
Duration of the disease (years) & $12(1-49)$ & $10(0.25-40)$ & 0.250 \\
Methotrexate (percentage of patients in use) & $17(33.33 \%)$ & $19(41.30 \%)$ & 0.417 \\
Prednisone (percentage of patients in use) & $9(17.65 \%)$ & $10(21.74 \%)$ & 0.612 \\
Sulfasalazine (percentage of patients in use) & $2(3.92 \%)$ & $3(6.52 \%)$ & 0.563 \\
Biologicals (percentage of patients in use) & $3(5.88 \%)$ & $2(4.35 \%)$ & 0.733 \\
\hline
\end{tabular}

Group PAO: Patients with psoriatic arthritis and onychodystrophy.; Group PO: Patients with cutaneous psoriasis and onychodystrophy.; Group PA: Patients with psoriatic arthritis and clinically normal nails.; Group P: Patients with cutaneous psoriasis and clinically normal nails.

The numerical variables are described with the median values and, in parentheses, the minimum and maximum values. The other variables are described in percentage.

TABLE 2: Microscopic variables of patients with and without onychodystrophy - results of the variables found in each group, separately, and comparison between groups

\begin{tabular}{|c|c|c|c|c|c|c|c|c|}
\hline & PO (31) & $\mathrm{P}(25)$ & PAO (20) & PA (21) & $\begin{array}{l}\mathrm{p} \text { value } \\
\text { POxP } \\
\text { Patients with } \\
\text { cutaneous } \\
\text { psoriasis }\end{array}$ & $\begin{array}{l}\text { p value } \\
\text { PAOxPA } \\
\text { Patients with } \\
\text { psoriatic } \\
\text { arthritis }\end{array}$ & $\begin{array}{l}\text { p value } \\
\text { POxPAO } \\
\text { Patients with } \\
\text { onicodystro- } \\
\text { phy }\end{array}$ & $\begin{array}{l}\text { p value } \\
\text { PxPA } \\
\text { Patients with } \\
\text { clinically } \\
\text { normal nails }\end{array}$ \\
\hline $\begin{array}{l}\text { Thickness of the } \\
\text { nail plate (mm) }\end{array}$ & $0.4(0.26-0.8)$ & $0.39(0.25-0.7)$ & $0.41(0.26-0.85)$ & $0.38(0.2-0.8)$ & 0.719 & 0.190 & 0.201 & 0.878 \\
\hline $\begin{array}{l}\text { Subungual thick- } \\
\text { ness (mm) }\end{array}$ & $0.2(0-0.7)$ & $0.1(0-0.3)$ & $0.1(0-0.4)$ & $0.1(0-0.55)$ & 0.002 & 0.510 & 0.017 & 0.431 \\
\hline $\begin{array}{l}\text { Layers of paraker- } \\
\text { atosis }\end{array}$ & $5(0-36)$ & $1(0-8)$ & $3.5(0-53)$ & $2(0-9)$ & 0.0006 & 0.461 & 0.383 & 0.245 \\
\hline Neutrophils & $4(12.90 \%)$ & $0(0 \%)$ & $2(10.00 \%)$ & $1(4.76 \%)$ & 0.086 & 0.481 & 0.561 & 0.456 \\
\hline Serum lakes & $17(54.84 \%)$ & $5(20 \%)$ & $7(35.00 \%)$ & $10(47.62 \%)$ & 0.008 & 0.412 & 0.166 & 0.047 \\
\hline Blood & $5(16.13 \%)$ & $1(4 \%)$ & $1(5.00 \%)$ & $1(4.76 \%)$ & 0.154 & 0.744 & 0.230 & 0.710 \\
\hline Fungi & $8(25.81 \%)$ & $2(8.00 \%)$ & $3(15.00 \%)$ & $3(14.29 \%)$ & 0.082 & 0.290 & 0.362 & 0.41 \\
\hline
\end{tabular}

Group PAO: Patients with psoriatic arthritis and onychodystrophy. ; Group PO: Patients with cutaneous psoriasis and onychodystrophy. ;Group PA: Patients with psoriatic arthritis and clinically normal nails.; Group P: Patients with cutaneous psoriasis and clinically normal nails.; PO $\mathbf{x}$ P: $\mathrm{p}$ value in the statistical comparison between the groups PO and P. ; PAO $\mathbf{x}$ PA: $\mathrm{p}$ value in the statistical comparison between the groups PAO and PA. ; PO $\times$ PAO: $p$ value in the statistical comparison between the groups PO and PAO; P $\times$ PA: $p$ value in the statistical comparison between the groups P and PA.

The numerical variables are described with the median values and, in parentheses, the minimum and maximum values. The other variables are described in percentage and, in parentheses, the absolute values. 
In Table 2, all the microscopic variables are exposed comparatively between the groups, in a global way.

\section{DISCUSSION}

Nail dystrophy is observed in $40 \%$ to $45 \%$ of patients with exclusively cutaneous psoriasis and in $87 \%$ of patients with psoriatic arthritis., ${ }^{3,15,16}$ Studies have shown that nail involvement is closely related to psoriatic arthritis and may represent a predictor of the disease. ${ }^{16-19}$

Patients with psoriatic arthritis have a greater reduction in quality of life and functional capacity when compared with patients with exclusively cutaneous psoriasis. Because of the appearance of cutaneous lesions earlier than in articular manifestations in $80 \%$ of patients, the search for indicators that aid in the early detection and treatment of joint symptoms in patients with arthropathic psoriasis is of great clinical interest. ${ }^{1,2,16,20}$ Several questionnaires for application in dermatological clinics have already been created in the search for an early detection of psoriatic arthritis. Recently, a questionnaire composed of four questions and a clinical nail analysis showed a sensitivity of $86.9 \%$ and specificity of $71.3 \%$ when three positive parameters were found..$^{20}$

In the search for a better understanding of the relation between exclusively cutaneous psoriasis, nail psoriasis and psoriatic arthritis, several hypotheses have been studied to try to clarify the etiopathogenesis of these multiple facets of psoriasis. One hypothesis is based on the possible existence of an autoantigen present in the skin, nail apparatus and synovial membranes, which would trigger a $\mathrm{T}$ lymphocyte-mediated inflammatory response in these three distinct anatomical locations. An autoantigen was well established in cutaneous psoriasis, through the identification of HLA-Cw6. Similarly, the inflammatory reaction mediated by $\mathrm{T}$ lymphocytes producing interleukins 12 and 23 has also been proven in patients with exclusively cutaneous disease. ${ }^{21-23}$ However, this model failed to explain the relation between disease involvement in the joints and nail disease, since common nail and joint autoantigens were not identified, as well as the association between interleukins and nail psoriasis and non-psoriatic arthritis is not clearly established..$^{21-23}$

Another theory to try to explain the greater relation between nail psoriasis and psoriatic arthritis is the anatomic connection between the nail structures and the distal interphalangeal joint. By means of high resolution magnetic resonance imaging, it is possible to identify that the fibers of the extensor tendon of the fingers are anchored directly in the nail matrix and in the periosteum of the distal phalanx, where the nail bed rests. ${ }^{6}$ Additionally, collateral ligaments are attached to the lateral edges of the nail, making the nail apparatus an integral part of the musculoskeletal system of the finger. ${ }^{16,21-23}$ Distal interphalangeal joint enthesitis could trigger the nail structure impairment leading to the onset of nail psoriasis. ${ }^{17,22,23} \mathrm{Al}$ though this interpretation is interesting when there is concomitance between nail and finger joints disease, it is of little use for cases of pure cutaneous psoriasis in which dystrophic nails exist even in the absence of arthritis and psoriatic plaques on the hands or feet, for example. A third explanation for onychodystrophy would be based on the fact that the chirodactiles represent frequent areas of trauma through the theory of the Koebner phenomenon. ${ }^{24}$
In the present study, NAPSI was applied as a method of clinical evaluation of the degree of nail involvement by the disease. There was no statistical difference in NAPSI values among patients with or without psoriatic arthritis. Palmou et al. obtained similar results, however, they identified a higher prevalence of pitting and splinter hemorrhage in those with exclusively cutaneous psoriasis. ${ }^{18}$ Other studies have demonstrated a greater nail involvement through NAPSI in patients with psoriatic arthritis, with onycholysis being prevalent in the group with joint disease. ${ }^{5,24}$ The methods chosen for evaluation by NAPSI vary among studies, and comparison of results should be conducted very cautiously. According to data from the literature and information obtained with the present study, therefore, it seems pertinent to state that onychodystrophy is more frequent in patients with psoriatic arthritis, but it is not necessarily more severe than that observed in patients with the exclusive cutaneous form. This data should be taken into account when discussing the etiopathogenesis of nail psoriasis, related or not to arthropathy.

Some clinical variables, however, were statistically different between the groups studied. There were significantly more women in the group with psoriatic arthritis and clinically normal nails. This finding does not have explanations in the literature, which indicates a similar affection in men and women, in both psoriasis and psoriatic arthritis, and may correspond to a selection bias. ${ }^{25}$ As for the medications used, as expected, systemic drugs were statistically prevalent in patients with psoriatic arthritis, as well as corticosteroids and sulfasalazine were more commonly used in patients with joint manifestations.

From the angle of the microscopic evaluation of the dystrophic nails, line of research presented for the first time in this article, cutaneous psoriasis and arthropathic psoriasis are very similar. Patients with onychodystrophy and exclusively cutaneous psoriasis exhibited nails with the subungual region thicker than the other groups, a statistically significant finding. The thickness of the nail plate, however, did not appear to be significantly different between the studied groups. The overall increase in nail thickness, with or without surface irregularities, is one of the main findings in magnetic resonance imaging of the nail..$^{26,27}$ Gisondi et al., in a nail evaluation by ultrasonography, documented a greater thickness of the nail plate and bed in patients with psoriasis compared with patients with eczema (even with normal nails) and controls. ${ }^{28}$

Layers of parakeratosis were present in greater numbers among patients with onychodystrophy, both with exclusively cutaneous psoriasis and those with arthritis. Neutrophils were found slightly more frequently in patients with onychodystrophy, but not in sufficient amplitude to become statistically relevant and independent of joint status, as previously mentioned. A patient with clinically normal arthritis and nails presented neutrophils on microscopic examination. Regarding serous lakes, in those individuals with clinically normal nails, there was higher statistical evidence in the psoriatic arthritis group, being observed in $48 \%$ of the patients, and in the group with psoriasis and onychodystrophy, it was present in $54 \%$. The fact that patients with cutaneous psoriasis or psoriatic arthritis have nails without visible clinical changes, but microscopically altered, is at least instigating. Prospective studies, however, would be 
needed to better assess the importance of this finding, especially to determine whether microscopic changes in clinically normal nails are related to the development/ severity of nail psoriasis or psoriatic arthritis in the future.

Our study has limitations that should be mentioned. The cross-sectional design did not allow us to evaluate longitudinal relations between clipping alterations and clinical evolution of the patients, such as the transition from cutaneous psoriasis to psoriatic arthritis. Frequent use of medications may have interfered with the degree of nail dystrophy and, therefore, the NAPSI score and the microscopic findings of the nails evaluated. Finally, a larger sample number could have contributed to a statistical analysis with more significant results.

\section{CONCLUSION}

Nail clipping represents a simple and quick method for diagnosis and assessment of nails of patients with nail psoriasis, with cutaneous and/or joint manifestations. With the clinical and microscopic results found in the present study, it appears that, once the nail apparatus is reached by psoriasis, the outcome is similar in the presence or absence of arthritis.]

\section{REFERENCES}

1. Cohen MR, Reda DJ, Clegg D0. Baseline relationships between psoriasis and psoriatic arthritis: analysis of 221 patients with active psoriatic arthritis. J Rheumatol. 1999;26:1752-6.

2. Ciocon $\mathrm{DH}$, Kimball $\mathrm{AB}$. Psoriasis and psoriatic arthritis: separate or one and the same? Br J Dermatol. 2007;157:850-60.

3. Baran R. The burden of nail psoriais: an introduction. Dermatology. 2010;221:1-5.

4. Romiti R. Compêndio de Psoríase. Rio de Janeiro: Elsevier; 2010.

5. Williamson L, Dalbeth N, Dockerty JL, Gee BC, Weatherall R, Wordsworth BP. Extended report: nail disease in psoriatic arthritis- clinically important, potentially treatable and often overlooked. Rheumatology (Oxford). 2004;43:790-4.

6. Tan AL, Benjamin M, Toumi H, Grainger AJ, Tanner SF, Emery P, et al. The relationship between the extensor tendon enthesis and the nail in distal interphalangeal joint disease in psoriatic arthritis- a high resolution MRI and histological study. Rheumatology (0xford). 2007;46:253-6.

7. Fillus Neto J, Tchornobay AM. Como o clipping pode auxiliar o dermatologista. An Bras Dermatol. 2009;84:173-6.

8. Wilsmann-Theis D, Sareika F, Bieber T, Schmid-Wendtner MH, Wenzel J. New reasons for histopathological nail-clipping examination in the diagnosis of onychomycosis. J Eur Acad Dermatol Venereol. 2011;25:235-7.

9. Lawry MA, Haneke E, Strobeck K, Martin S, Zimmer B, Romano PS. Methods for diagnosing onychomycosis: a comparative study and review of the literature. Arch Dermatol. 2000;136:1112-6.

10. Werner B, Fonseca GP, Seidel G. Microscopic nail clipping findings in patients with psoriasis. Am J Dermatopathol. 2015;37:429-39

11. Stephen S, Tosti A, Rubin Al. Diagnostic applications of nail clipping. Dermatol Clin. 2015;33:289-301.

12. Rich P, Scher RK. Nail Psoriasis Severity Index: a useful tool for evaluation of nail psoriasis. J Am Acad Dermatol. 2003;49:206-12.

13. Parrish CA, Sobera J0, Elewski BE. Modifications of the nail psoriasis severity index (letter). J Am Acad Dermatol. 2005;53:745-6

14. Tillett W, Costa L, Jadon D, Wallis D, Cavill C, McHugh J J et al. The Classification of psoriatic arthritis (CASPAR) criteria- a retrospective feasibility, sensitivity and specificity study. J Rheumatol. 2012;39:154-6.

15. Werner B, Antunes A. Microscopic examination of normal nail clippings. Dermatol Pract Concept. 2013;3:9-14.

16. Langenbruch A, Radtke MA, Krensel M, Jacobi A, Reich K, Augustin M. Nail involvement as a predictor of concomitant psoriatic arthritis in patients with psoriasis. Br J Dermatol. 2014;171:1123-8.

17. McGonagle D, Tan AL, Benjamin M. The nail as a musculoskeletal appendageimplications for an improved understanding of the link between psoriasis and arthritis. Dermatology. 2009;218:97-102.

18. Palmou N, Marzo-Ortega H, Ash Z, Goodfield M, Coates LC, Helliwell PS, et al . Linear pitting and splinter haemorrhages are more commonly seen in the nails of patients with established psoriasis in comparison to psoriatic arthrits. Dermatology. 2011;223:370-3

19. Wilson FC, Icen M, Crowson CS, McEvoy MT, Gabriel SE, Kremers HM. Incidence and clinical predictors of psoriatic arthritis in patients with psoriasis: a populationbased study. Arthritis Rheum. 2009;61:233-9.

20. Garg N, Truong B, Ku JH, Devere TS, Ehst BD, Blauvelt A, et al. A novel, short and simple screening questionnaire can suggest presence of psoriatic arthritis in psoriasis patients in a dermatologic clinic. Clin Rheumatol. 2015;34:1745-51.

21. McGonagle D, Benjamin M, Tan AL. The pathogenesis of psoriatic arthritis and associated nail disease: not autoimmune after all. Curr Opin Rheumatol. 2009;21:340-7.

22. McGonagle D, Palmou Fontana N, Tan AL, Benjamin M. Nailing down the genetic and immunological basis for psoriatic disease. Dermatology. 2010;221:15-22.

23. McGonagle D. Enthesitis: an autoinflammatory lesion linking nail and join involvement in psoriatic disease. J Eur Acad Dermatol Venereol. 2009;23:9-13

24. Maejima H, Taniguchi T, Watarai A, Katsuoka K. Evaluation of nail disease in psoriatic arthritis by using a modified nail psoriasis severity score índex. Int J Dermatol. 2010;49:901-6.

25. Bologna JL, Jorizzo JL, Rapini RP. Dermatology. 2nd ed. Spain: Mosby Elsevier; 2008.

26. Soscia E, Sirignano C, Catalano 0, Atteno M, Costa L, Caso F, et al. New developments in magnetic resonance imaging of the nail unit. J Rheumatol Suppl. 2012;89:49-53.

27. Scarpa R, Soscia E, Peluso R, Atteno M, Manguso F, Del Puente A, et al. Nail and distal interphalangeal joint in psoriatic arthritis. J Rheumatol. 2006;33:1315-9.

28. Gisondi P, Idolazzi L, Girolomoni G. Ultrasonography reveals nail thickening in patients with chronic plaque psoriasis. Arch Dermatol Res. 2012;304:727-32.
MAILING ADDRESS:
Gabriela Poglia Fonseca
R. General Carneiro, 181
Alto da Glória
80060-900 - Curitiba, PR
Brazil
Email: gabipoglia@hotmail.com

How to cite this article: Fonseca GP, Werner B, Seidel G, Staub HL. Comparative microscopic analysis of nail clippings from patients with cutaneous psoriasis and psoriatic arthritis. An Bras Dermatol. 2017;92(1):21-5. 